\title{
Bose-Einstein Condensation with Attractive Boundary Conditions
}

\author{
Derek W. Robinson \\ Département de Physique, Université d'Aix-Marseille II, Luminy, and Centre de Physique Théorique, \\ CNRS, F-13274 Marseille Cedex 2*, France
}

\begin{abstract}
The phenomena of Bose-Einstein condensation is discussed for particles in a box with attractive walls. Variation of the elasticity has the following effects, a) the critical temperature, fugacity, etc. vary, b) separation of phases occurs, c) condensation in one and two dimensions is possible.
\end{abstract}

\section{Introduction}

Theoretical understanding of phase transitions has been greatly enhanced by the study of simple soluble models such as the Ising model or the ideal Bose gas. The spontaneous magnetization exhibited by the former model has been examined by variation of boundary conditions (for a review see [1]). In two dimensions this model appears to have the simple feature that its basic thermodynamic structure is not affected by such variation. The boundary conditions only affect the relative proportions of the phases. This simplification is probably not shared by the three-dimensional model and is certainly not valid for the ideal Bose gas.

Condensation of the Bose gas has been exhaustively studied with periodic boundary conditions or Neumann conditions, $\partial \psi=0$ (see, for example, [2-4]). We consider a family of conditions, $\partial \psi=\sigma \psi$, which correspond to varying the elasticity of the walls containing the system and examine the influence of these conditions on the condensation phenomena ${ }^{1}$. The effects are threefold if the walls are attractive;

1. The thermodynamic region, the critical temperature, etc. vary with the elasticity and, in particular, condensation occurs at values of the fugacity, $z$, strictly less than one.

2. Phase separation occurs.

3. Condensation occurs in one and two dimensions.

Each of these effects is illustrated by examination of the density of the system.

* Postal address

1 In [4] the conditions $\partial \psi=a \psi$ are considered for a system of dimension $L$ but with $a \sim L^{-1}$ and hence for macroscopic systems this is effectively $\partial \psi=0$ 


\section{Elastic Boundary Conditions}

Consider a system of particles confined to a cubic box whose edges are of length $L$. The various directional degrees of freedom of each particle are independent and hence the multiparticle, multi-dimension, eigenvalues and eigenfunctions of the system are obtained by combination of the corresponding quantities evaluated for one particle in one dimension. Initially it suffices to consider the eigenvalue equation

$$
-d^{2} \psi_{\lambda}(x) / d x^{2}=\lambda \psi_{\lambda}(x)
$$

for $\psi_{\lambda} \in C^{2}(0, L)$ where we have chosen units such that $\hbar^{2} / 2 m=1$. The elastic boundary conditions form a one-parameter family of restraints

$$
\left[d \psi_{\lambda}(x) / d x-\sigma \psi_{\lambda}(x)\right]_{x=0}=0=\left[d \psi_{\lambda}(x) / d x+\sigma \psi_{\lambda}(x)\right]_{x=L} .
$$

The choice

$$
\psi_{\lambda}(x)=\operatorname{Cos} \sqrt{\lambda} x+\sigma \operatorname{Sin} \sqrt{\lambda} x / \sqrt{\lambda}
$$

solves (1) and (2) gives the eigenvalue equation

$$
\operatorname{Tan} \sqrt{\lambda} L / \sqrt{\lambda}=2 \sigma /\left(\lambda-\sigma^{2}\right) .
$$

Real, or imaginary, values are allowed for $\sqrt{\lambda}$, i.e. positive, or negative, values of $\lambda$.

Equation (4) can be solved by graphical techniques (for details see, for example, [5]). The eigenvalues $\left\{\lambda_{n}(\sigma, L)\right\}_{n \geqq 0}$ are monotonically increasing functions of $\sigma$ for fixed $L$ with the following properties. If $\sigma<-2 / L$ there are two negative eigenvalues and

$$
\lambda_{0}<-\sigma^{2}<\lambda_{1}<0, \quad(\pi / L)^{2}<\lambda_{2}<(2 \pi / L)^{2}<\lambda_{3}<\ldots .
$$

If $-2 / L<\sigma<0$ then there is one negative eigenvalue

$$
\lambda_{0}<-\sigma^{2}, \quad 0<\lambda_{1}<(\pi / L)^{2}<\lambda_{2}<(2 \pi / L)^{2}<\ldots .
$$

If $\sigma>0$ then there are no negative eigenvalues and

$$
0<\lambda_{0}<(\pi / L)^{2}<\lambda_{1}<(2 \pi / L)^{2}<\lambda_{2} .
$$

There are two simple cases, $\sigma=0$, or Neumann conditions, for which

$$
\lambda_{n}(0, L)=(n \pi / L)^{2}, \quad n=0,1,2, \ldots
$$

and the limiting case $\sigma \rightarrow+\infty$, or Dirichlet boundary conditions for which

$$
\lambda_{n}(\infty, L)=((n+1) \pi / L)^{2}, \quad n=0,1,2, \ldots .
$$

The behaviour of the eigenvalues as a function of $L$ with $\sigma$ fixed is also relevant. All positive eigenvalues decrease monotonically to zero. If, however, $\sigma \leqq 0$ then

$$
\lim _{L \rightarrow \infty} \lambda_{0}(\sigma, L)=-\sigma^{2}=\lim _{L \rightarrow \infty} \lambda_{1}\left(\sigma_{1} L\right)
$$

where the first limit is monotonic upwards and the second monotonic downwards and in fact one has

$$
\begin{aligned}
& \lambda_{0}(\sigma, L)=-\sigma^{2}+O\left(e^{-|\sigma| L}\right) \\
& \lambda_{1}(\sigma, L)=-\sigma^{2}+O\left(e^{-|\sigma| L}\right) .
\end{aligned}
$$


The corresponding unnormalized eigenfunctions are approximately given by

$$
\psi_{0}(x)=\operatorname{Ch} \sigma(x-L / 2), \quad \psi_{1}(x)=\operatorname{Sh} \sigma(x-L / 2) .
$$

For example $\psi_{0}$ satisfies the eigenvalue Equation (1) and for (2) one finds

$$
\left[\psi_{0}(x)^{-1} d \psi_{0}(x) / d x-\sigma\right]_{x=0}=O\left(e^{-|\sigma| L / 2}\right)=\left[\psi_{0}(x)^{-1} d \psi_{0}(x) / d x+\sigma\right]_{x=L} .
$$

The negative eigenvalues correspond physically to states in which the particle is bound to the walls. The eigenfunctions (5), when normalized, assign an insignificant probability that the particle is at a distance greater than $2 /|\sigma|$ from either endpoit of the interval $[0, L]$. Thus $\sigma<0$ conditions correspond to attractive walls.

The Neumann boundary conditions, $\sigma=0$, correspond to perfectly elastic walls; the ground state probability distribution is constant throughout the interval. The $\sigma>0$ conditions correspond to repulsive boundaries; the ground state wavefunctions assign small probability to finding the particle near the endpoints $x=0$, or $x=L$.

The corresponding eigenvalues of a particle in a $v$-dimensional cubic box $\Lambda$ with eigenfunctions satisfying

$$
\partial \psi(x) / \partial n=\sigma \psi(x), \quad x \in \partial \Lambda
$$

where $\partial / \partial n$ denotes the inward normal derivative are given by

$$
\lambda_{\boldsymbol{n}}(\sigma, L)=\sum_{i=1}^{v} \lambda_{n_{t}}(\sigma, L)
$$

and the components $n_{i}$, of the vector $\boldsymbol{n}$, take the values $n_{i}=0,1,2, \ldots$.

\section{Bose-Einstein Condensation}

Our discussion of Bose-Einstein condensation is limited for brevity to an analysis of the particle density in the grand canonical ensemble. Recall that the grand canonical density in $v$-dimensions, $\varrho_{L}^{(v)}$, is defined as a function of the inverse temperature, $\beta$, and the fugacity, $z$, by

$$
\varrho_{L}^{(\nu)}(\beta, z)=L^{-v} \sum_{\boldsymbol{n}} z e^{-\beta \lambda_{\boldsymbol{n}}(\sigma, L)} /\left(1-z e^{-\beta \lambda_{\boldsymbol{n}}(\sigma, L)}\right)
$$

where $\lambda_{n}$ are given by (6) and this expression is valid for $0 \leqq z<\exp \left\{-\beta v \lambda_{0}\right\}$. We analyze this function for various values of $\sigma$ and various dimensions $v$. The variation of dimension is because the properties of $\varrho_{L}^{(2)}, \varrho_{L}^{(1)}$, are of use in the analysis of $\varrho_{L}^{(3)}$.

\section{a) Dirichlet Boundary Conditions, $\sigma=+\infty$}

The Dirichlet density $\varrho_{L}^{(v)}$ is defined for $0 \leqq z<\exp \left\{-\beta v(\pi / L)^{2}\right\}$. In particular the interval $0 \leqq z \leqq 1$ is allowed for all $L<+\infty$. On this interval

$$
\begin{aligned}
\int_{n_{l} \geqq x_{i} \geqq n_{\imath}-1} d^{v} x z e^{-a x^{2}} /\left(1-z e^{-a x^{2}}\right) & \geqq z e^{-a \boldsymbol{n}^{2}} /\left(1-z e^{-a \mathbf{n}^{2}}\right) \\
& \geqq \int_{n_{i}+1 \geqq x_{i} \geqq n_{l}} d^{v} x z e^{-a x^{2}} /\left(1-z e^{-a x^{2}}\right), \quad a>0 .
\end{aligned}
$$


Thus with $\lambda=\pi \sqrt{\beta}$ summation and a simple change of variable yields

$$
\begin{aligned}
\lambda^{-v} \int_{x_{i} \geqq 0} d^{v} x z e^{-x^{2}} /\left(1-z e^{-x^{2}}\right) & \geqq \varrho_{L}^{(v)}(\beta, z) \\
& \geqq \lambda^{-v} \int_{x_{1} \geqq \lambda / L} d^{v} x z e^{-x^{2}} /\left(1-z e^{-x^{2}}\right) .
\end{aligned}
$$

Introducing the notation

$$
I_{v}(z)=\int d^{v} x z e^{-x^{2}} /\left(1-z e^{-x^{2}}\right), \quad I_{0}(z)=z /(1-z)
$$

one easily estimates that

$$
0 \leqq I_{v}(z)-\lambda^{v} \varrho_{L}^{(v)}(\beta, z) \leqq v J_{v}(z, L)
$$

where

$$
J_{v}(z, L)=\int_{0}^{\lambda / L} d y I_{v-1}\left(z e^{-y^{2}}\right) .
$$

Note that $z \in[0,1] \mapsto I_{v}(z) \in \mathbb{R}$ is positive, increasing, concave. Further $I_{v}(1)<+\infty$ for $v \geqq 3$ but $I_{1}$ and $I_{2}$ diverge at $z=1$. The $J_{v}$ have similar properties. Upper bounds for the $I_{v}$ and $J_{v}$ can be calculated by elementary means and for example one has for $0 \leqq z \leqq 1$

$$
\begin{array}{ll}
I_{3}(z) \leqq I_{3}(1)<3, & J_{3}(z, L) \leqq 2\left[-(\lambda / L) \log (\lambda / L)+(\lambda / L)+(1 / 6)(\lambda / L)^{3}\right] \\
I_{2}(z) \leqq \log 1 /(1-z), & J_{2}(z, L) \leqq 2(\lambda / L) z /(1-z)^{1 / 2} \\
I_{1}(z) \leqq 2 z /(1-z)^{1 / 2}, & J_{1}(z, L) \leqq z /(1-z) .
\end{array}
$$

Combination of (8) and (9) with the estimates (10) establish that

$$
\lim _{L \rightarrow \infty} \varrho_{L}^{(v)}(\beta, z)=\lambda^{-v} I_{v}(z), \quad v \leqq 3,0 \leqq z \leqq 1 .
$$

More precisely one has

$$
0 \leqq I_{\nu}(z)-\lambda^{v} \varrho_{L}^{(v)}(\beta, z) \leqq O((\lambda / L) \log (\lambda / L))
$$

for $v=3$ and $0 \leqq z \leqq 1$, or for $v=1,2$, and $0 \leqq z<1$.

Thus $0 \leqq \lambda^{3} \varrho_{L}^{(3)}(\beta, z) \leqq I_{3}(1)$ and there is an innate restriction on the simultaneous values of $\varrho_{L}^{(3)}$ and $\beta$. No such restrictions exist if $v=1,2$, because of the divergence of $I_{1}$ and $I_{2}$ at $z=1$. Referring to the standard interpretation $[2,3]$ we conclude that Dirichlet conditions lead to the uncondensed phase for $v=3$ and the conventional Bose-Einstein condensation is incompatible with such conditions.

(Note that the parameter $\lambda$ can be essentially identified with the thermal wavelength $v=\sqrt{2 \pi \beta \hbar^{2} / m}$. With our choice of units $v=2 \lambda / \sqrt{\pi} \approx 1$. $1 \lambda$. This identification shows that the accuracy of approximation of $\varrho_{L}^{(v)}$ by its infinite volume limit is remarkable. Even at unfavourably low temperatures of $1^{\circ} \sim 2^{\circ} \mathrm{K}$, with $L=1 \mathrm{~cm}$, and for light atoms, the approximation is valid to about one part in $10^{5}$.)

\section{b) Neumann Conditions, $\sigma=0$}

For Neumann conditions the density (7) is defined for all $0 \leqq z<1$ but the value $z=1$ is not allowed. The eigenvalues given by these boundary conditions differ 
from the Dirichlet conditions insofar that extra zero energy modes occur. This allows the decomposition of the Neumann density as a sum of Dirichlet densities and then the estimates of the previous subsection can be applied. Let $\tau_{L}^{(v)}$ denote the Neumann density then one has

$$
\tau_{L}^{(v)}(\beta, z)=\sum_{m=0}^{v-1} L^{-m}\left(\begin{array}{l}
v \\
m
\end{array}\right) \varrho_{L}^{(v-m)}(\beta, z)+L^{-v} z /(1-z)
$$

which gives

$$
\begin{aligned}
0 & \leqq \lambda^{v} \tau_{L}^{(v)}(\beta, z)-(\lambda / L)^{v} z /(1-z)-\lambda^{v} \varrho_{L}^{(v)}(\beta, z) \\
& =\sum_{m=1}^{v-1}(\lambda / L)^{m}\left(\begin{array}{c}
v \\
m
\end{array}\right) \lambda^{v-m} \varrho_{L}^{(v-m)}(\beta, z) \leqq \sum_{m=1}^{v-1}(\lambda / L)^{m}\left(\begin{array}{l}
v \\
m
\end{array}\right) I_{v-m}(\beta, z) .
\end{aligned}
$$

If $z<1$ then

$$
0 \leqq \lambda^{\nu} \tau_{L}^{(\nu)}(\beta, z)-\lambda^{v} \varrho_{L}^{(\nu)}(\beta, z)=O(\lambda / L)
$$

and the difference between the Dirichlet and Neumann densities is negligible. In particular $\lambda^{3} \tau_{L}^{(3)}(\beta, z)$ is bounded. Nevertheless the density can be increased above the previous maximum by choosing $z$ sufficiently close to unity where sufficiently close means that the value of $z$ must be chosen as a function of the size of the system. For $\varrho_{0}>0$ choose $z=z_{L}=1-1 / \varrho_{0} L^{3}$ and then

$$
0 \leqq \lambda^{3} \tau_{L}^{(3)}(\beta, z)-\lambda^{3} \varrho_{0} z_{L}-\lambda^{3} \varrho_{L}^{(3)}(\beta, z) \leqq 3 \sum_{m=1}^{2}(\lambda / L)^{m} J_{3-m}(\beta, z) .
$$

Using the relations

$$
0 \leqq I_{v}(1)-I_{v}(z) \leqq(1-z) I_{v}(1), \quad v \geqq 3
$$

and the estimates for $I_{1}$ and $I_{2}$ one has

$$
\begin{aligned}
-(\lambda / L)\left[1+I_{3}(1) / \varrho_{0}\right] & \leqq \lambda^{3}\left[\tau_{L}^{(3)}\left(\beta, z_{L}\right)-\varrho_{0}-\varrho_{L}^{(3)}(\beta, 1)\right] \\
& \leqq 6(\lambda / L)^{1 / 2}\left(\lambda^{3} \varrho_{0}\right)^{1 / 2}-9\left(\lambda^{3} \varrho_{0}\right)^{-1 / 3}(\lambda / L) \log (\lambda / L) .
\end{aligned}
$$

This establishes the precise estimate

$$
\lambda^{3} \tau_{L}^{(3)}(\beta, z)-\left[\lambda^{3} \varrho_{0}+I_{3}(1)\right]=O\left((\lambda / L)^{1 / 2}\right)
$$

for the condensation phenomena, i.e. the non-zero contribution to the density arising from the zero energy mode.

If $v=1,2$ the situation is different. The divergence of $I_{1}$ and $I_{2}$ at $z=1$ implies that the contribution of the zero energy mode obtained by the above mechanism is negligible. Thus Bose-Einstein condensation is absent for $v=1,2,[6]$, when Neumann boundary conditions are used.

\section{c) Attractive Walls, $\sigma<0$}

When $\sigma<0$ and $2<|\sigma| L$ there are two negative energy modes $\lambda_{0}=\lambda_{0}(\sigma, L)$, $\lambda_{1}=\lambda_{1}(\sigma, L)$, which contribute and the density is defined in the range of fugacity $0 \leqq z<\exp \left\{v \beta \lambda_{0}\right\}$. We now follow the method of the previous subsection and decompose the density into a sum of terms chosen according to the relative con- 


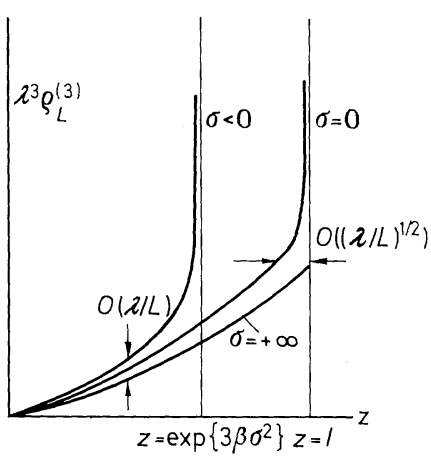

Fig. 1

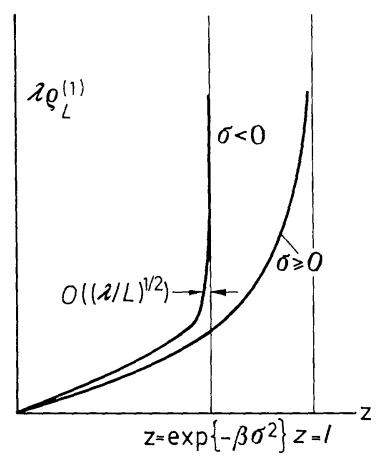

Fig. 2

tribution of $\lambda_{0}, \lambda_{1}$. Introduce the notation

$$
z_{i_{1} i_{2} \ldots i_{n}}=z e^{-\beta \lambda_{2}} \ldots e^{-\beta \lambda_{l n}}
$$

where $i_{p}$ is 0 or 1 . Let $\eta_{L}^{(v)}$ denote the $v$-dimensional density with $\sigma<0$ then

$$
\begin{aligned}
\eta_{L}^{(3)}(\beta, z)= & \sum_{i, j, k=0}^{1} L^{-3} z_{i j k}\left(1-z_{i j k}\right)+\sum_{i, j=0}^{1} \hat{\varrho}_{L}^{(1)}\left(\beta, z_{i j}\right) \\
& +\sum_{i=0}^{1} \hat{\varrho}_{L}^{(2)}\left(\beta, z_{i}\right)+\hat{\varrho}_{L}^{(3)}(\beta, z)
\end{aligned}
$$

where $\hat{\varrho}_{L}^{(v)}$ is defined by (7) but the components $n_{i}$ are summed only over the values $n_{i} \geqq 2$. As the eigenvalues $\lambda_{n}=\lambda_{n}(\sigma, L)$ satisfy $((n-1) \pi / L)^{2} \leqq \lambda_{n} \leqq(n \pi / L)^{2}$ for $n \geqq 2$ the $\varrho_{L}^{(v)}$ can be bounded in the same manner that we bounded the Dirichlet densities. One has

$$
0 \leqq I_{v}(z)-\lambda^{v} \hat{\varrho}_{L}^{(v)}(\beta, z) \leqq v J_{v}(z, L / 2) .
$$

Next we emulate the method used to exhibit condensation in the previous section. For $\nu=3$ choose $z=z_{L}=\left(1-8 / \varrho_{0} L^{3}\right) \exp \left\{3 \beta \lambda_{0}(\sigma, L)\right\}$ then by calculations of an identical nature to those used above one finds

$$
\left[\lambda^{3} \eta_{L}^{(3)}\left(\beta, z_{L}\right)-\lambda^{3} \varrho_{0}-I_{3}\left(e^{-3 \beta \sigma^{2}}\right)\right]=O\left((\lambda / L)^{1 / 2}\right)
$$

where the crucial point is that $\lambda_{0}(\sigma, L) \rightarrow-\sigma^{2}$ and $\lambda_{1}(\sigma, L) \rightarrow-\sigma^{2}$ as $L \rightarrow \infty$. Thus the picture of condensation is changed. For $\sigma<0$ Bose-Einstein condensation occurs for $z=\hat{z}_{\sigma}=\exp \left\{-3 \beta \sigma^{2}\right\}$ in three dimensions. The same effect also occurs in higher or lower dimensions with the critical fugacity $\hat{z}_{\sigma}=\exp \left\{-\beta v \sigma^{2}\right\}$ in $v$-dimensions.

To illustrate our conclusions we plot $\lambda^{3} \varrho_{L}^{(3)}(\beta, z)$ as a function $z$, for the various cases we have considered, in Figures 1 and 2. Figure 1 exhibits the behaviour typical of three or more dimensions and Figure 2 illustrates the behaviour for $v=1$, or 2 .

We conclude by remarking that the condensate in the $\sigma<0$ case consists of particles in the eigenstates which correspond to binding with the walls. Thus 
there is a separation of the phases with the condensed phase effectively concentrated within a distance $2 /|\sigma|$ from the walls of the system. The condensate, which is composed of approximately $\varrho_{0} L^{v}$ particles, occupies a volume approximately equal to $2^{v+1} L^{v-1} /|\sigma|$. Hence the density of the pure condensate is of the order $|\sigma| \varrho_{o} L$, i.e. the condensate has essentially infinite density and occupies essentially zero volume.

Acknowledgements. The material contained in this note is extracted from lectures to students of the "Maîtrise de Physique" at Luminy during the period 1969-1973; the author was persuaded by I. M. Sukov that publication could serve a wider pedagogical service. The final manuscript was prepared during a visit to the Summer Research Institute at the University of Adelaide. The author is indebted to A. Hurst for making this visit possible.

\section{References}

1. Gallavotti,G.: Riv. Nuovo Cimento 2, 133 (1972)

2. Huang, K.: Statistical Mechanics. New York-London: J. Wiley 1963

3. Landau, L., Lifschitz, E. : Statistical Physics. London: Pergamon 1958

4. Lewis, J., Pulè, J. V.: Commun. math. Phys. 36, 1 (1974)

5. Robinson, D.W.: The thermodynamic pressure in quantum statistical mechanics, Lecture Notes in Physics, Vol. 9. Berlin-Heidelberg-New York: Springer

6. Hohenberg, P.: Phys. Rev. 158, 383 (1967)

Communicated by G. Gallavotti

Received March 1, 1976 
